\title{
Primer registro de Abrothrix jelskii (Thomas 1824) para Chile
}

\section{First record of Abrothrix jelskii (Thomas 1894) for Chile}

\author{
Pablo Valladares Faúndez ${ }^{1 *}$, Nicole Álvarez Henríquez ${ }^{2}$, Natalia Urrutia ${ }^{2}$, Carlos Nassar San \\ MARTÍN $^{3}, \&$ Agustín IRIARTE ${ }^{4}$ \\ 1'Departamento de Biología, Facultad de Ciencias, Universidad de Tarapacá. Avenida General Velásquez 1775, Arica, Chile. \\ 2Universidad de La República, Rafael Sotomayor 420, Arica \\ ${ }^{3}$ Jefe Departamento de Áreas Silvestres Protegidas, Corporación Nacional Forestal. Vicuña Mackenna 820, Arica XV Región. \\ ${ }^{4}$ Flora \& Fauna Chile Ltda. Antonio Varas 175, Depto. 1009, Providencia, Santiago, Chile \\ *pvalladares@uta.cl
}

\begin{abstract}
Abrothrix jelskii (Thomas 1894) es una de las especies menos conocidas de su amplio género (Jayat et al. 2013). Su distribución ha sido registrada para los Andes del Sur de Perú (Smith \& Patton 1991; Salazar-Bravo et al. 2003; Pacheco et al. 2009), Bolivia (Tarifa \& Yensen 2001; Martínez et al. 2009; Anderson 1997) y el Noroeste de Argentina (Galliari et al. 1996; Teta et al. 2006; Díaz \& Bárquez 2007; Ferro 2010; Jayat et al. 2013). Su posición biogeográfica hacía suponer su presencia en Chile, pero hasta ahora no se había registrado en este país.
\end{abstract}

En este trabajo damos cuenta del primer registro de esta especie en Chile, con una colecta realizada el 03 de Mayo del 2014, de un espécimen de $A$. jelskii en el sector de Las Cuevas (18 $10^{\prime} 04,46^{\prime \prime} \mathrm{S}$; 69²5'48,94' 'O), en la Región de Arica y Parinacota, al extremo norte de Chile, en un ambiente altiplánico a 4479 msnm (Figura 1a), hábitat que se caracteriza por requeríos que bordean un bofedal (Figura 1b). Este nuevo hallazgo extiende el rango de distribución al poniente en 152 kilómetros desde Lipez, Bolivia, siendo la colecta más cercana al sector de Las Cuevas, en la Provincia de Parinacota, Chile. Existe un registro fotográfico previo de Roberto Tancara Querquezana, Guardaparque de la Corporación Nacional Forestal (CONAF) quién tomó la fotografía en el Parque Nacional Lauca $\left(17^{\circ} 57^{\prime} 51.41^{\prime \prime} \mathrm{S}\right.$, $\left.68^{\circ} 36^{\prime} 43.59^{\prime \prime} \mathrm{W}\right)$, a 32 kilómetros al oriente de la localidad de Las Cuevas.

Se colectó un ejemplar, que fue depositado en la Colección Zoológica de Zonas Áridas y Andinas (CZZA030) de la Universidad de Tarapacá, y corresponde a un macho adulto (Figura 2a), de tamaño mediano, con pelaje dorsal café con base de los pelos de color negro, orejas de tamaño mediano, con pelos rojizos brillantes, bordeados posteriormente con manchas blancas las que se mantienen separadas en la región occipital y que no se extienden hacia las mejillas. Zona del hélix de las orejas con pelos blancos. Nariz puntiaguda, con su extremo anterior rojizo brillante, similar al de las orejas, el que cubre todo el hocico, vibrisas mistaciales largas, y vibrisas supraorbitales y mentales de largo mediano. Zona parietal, temporal, frontal y nasal de color café, similar a la parte dorsal del cuerpo, color que llega hasta la zona media de la cabeza, en la región infraorbital y zygomática. Las subregiones maxilar, molar, mandibular y masetérica y garganta son de color blanco, la que se extiende por todo el largo ventral del cuerpo, con base de los pelos de color negro o gris oscuro. Dorso de las extremidades anteriores de color café lechoso claro, acompañada de pelos blancos y largos, con uñas muy largas. Manos con cinco dedos y uñas largas, pulgar pequeño acompañado de un gran cojinete carnoso. Palmar con tres almohadillas anteriores pequeñas y redondeadas, y uno posterior o carpal del doble del tamaño que los anteriores (Figura 2b). Extremidad posterior larga, pie largo, dorsalmente con pelos de color rojizo similares al hocico, plantar con tres almohadillas anteriores redondeados y pequeños, y tres posteriores de similar tamaño (Figura 2c). Cinco dedos con uñas largas. Cola más corta que el largo del cuerpo ( 0,85 veces), cubierta de pelos cortos de color rojizo en el dorso, y dividida con una franja delgada anteroposterior de color negro, zona ventral de la cola blanca. En relación al cráneo, nuestro ejemplar coincide con las descripciones de Jayat et al. (2013), excepto en el largo del hocico, que consideramos medianamente largo. De acuerdo a Sanborn (1947), esta población corresponde a la subespecie $A . j$. ochrotis por presentar orejas, pies, cola y nariz de color rojizo, es una subespecie con un morfo de color más oscuro en el dorso, blanco en el vientre y parches postauriculares bien definidos. La Terra typica de esta subespecie es la localidad de Huacullani, en el Departamento de Puno, Perú (Sanborn 1947) a $167 \mathrm{~km}$ del sector de Las Cuevas, lugar donde hacemos el primer registro de captura de esta especie en Chile.

Tuvimos un bajo esfuerzo de muestreo, con 300 trampas/ noche, usando trampas Sherman y Tomahawk. Junto con A. jelskii, fueron colectados dos ejemplares de Andinomys 
edax, un Auslyscomys boliviensis y una Lagidium peruanum. De acuerdo a las colecciones taxonómicas de referencia científica de Chile, $A$. edax, ha sido previamente registrada en el altiplano de la Región de Arica y Parinacota, en la localidad de Belén (18²9'S; 69³1'O; IEEUACH 7022), Murmuntani (18²1'08,99'S; 69³3'48.56”O; LCM 243) y Pampa Yuscuni (sin coordenadas; LCM 677, 678), mientras que $A$. boliviensis ha sido colectado en Parinacota (18¹8'47,95'S; 69²3'00,44'”O; PUCSSUC 9849; LCM194, 203, 588, 594), Las Cuevas (18¹0’04,46”'s;
69²5'48,94'”; LCM 1080, 1294) y Lago Chungará (18¹4'07,74's; 69¹0'57,78'O; PUCSSUC 9850; LCM 2044), y L. peruanum observada en gran abundancia en Las Cuevas (18 10'04,46'S; 69²5'48,94'O), Choquelimpie (18¹9'30.61"S; 69¹6’37.94'O) y Parinacota (18¹8'47,95'S; 69²3'00,44"O; LCM 609, 610, 658, 679). De todas las especies de roedores identificados para el altiplano de esta región, en esta oportunidad nosotros colectamos el $17,6 \%$.

Este primer registro de $A$. jelskii deja en evidencia la
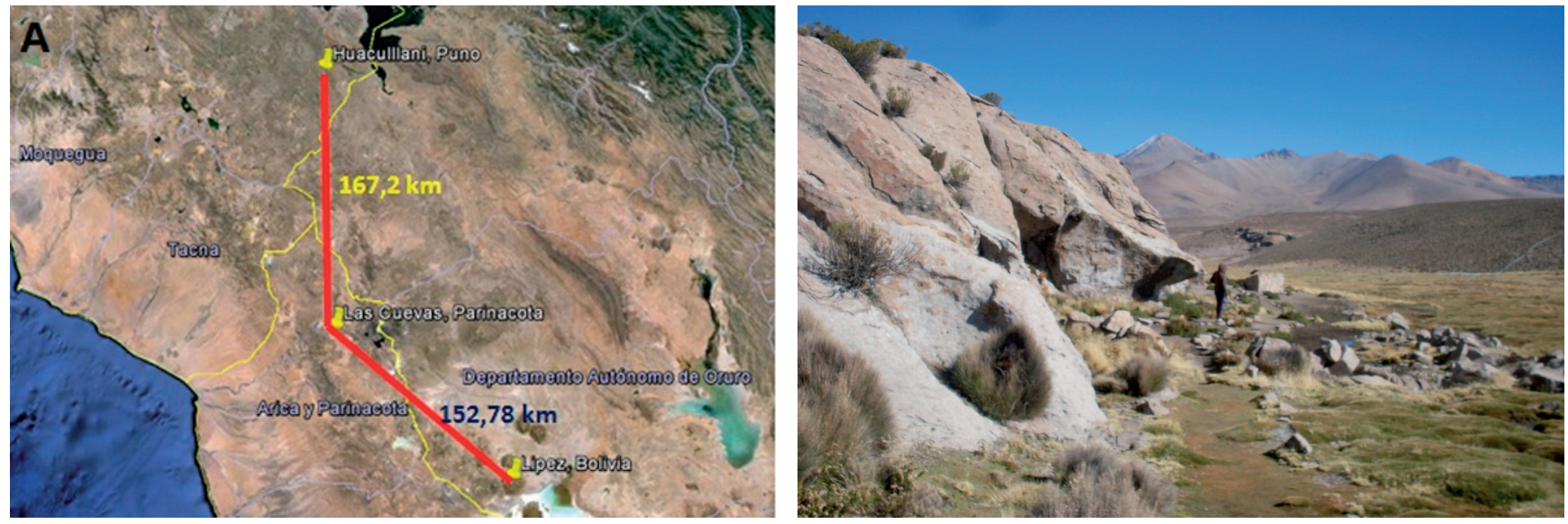

Figura 1. A: Nueva colecta de $A$. jelskii en Chile y distancia (en Kms) entre los puntos más próximos en Perú y Bolivia. B: Hábitat que corresponde a roqueríos bordeando un bofedal.

Figure 1. A: New collection of A. jelskii in Chile and distance (in kilometers) between the nearest points from Peru and Bolivia. B: Habitat corresponding to rocky bordering a swamp.
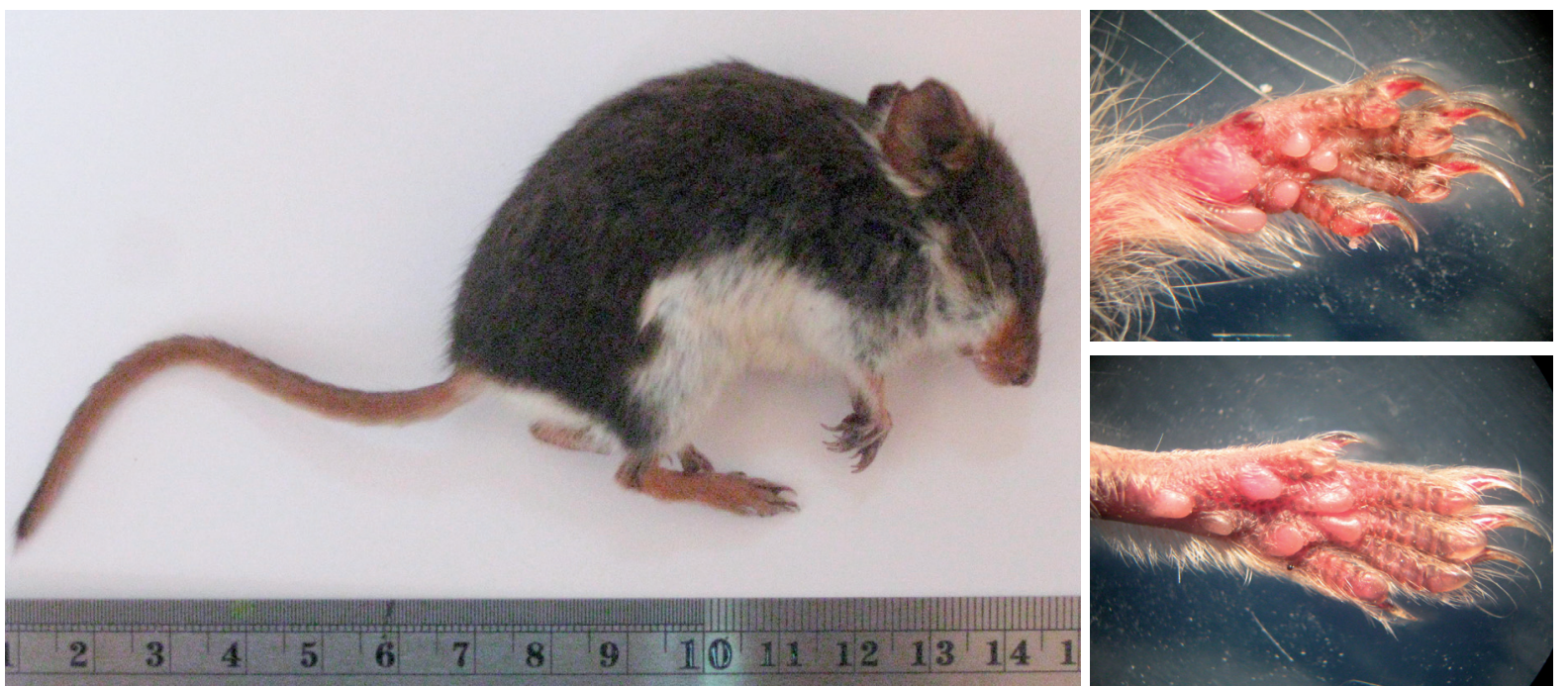

Figura 2. A: Primer espécimen de A. jelskii colectado en Chile, que corresponde a un macho adulto (CZZA030). B: Mano izquierda con cinco dedos, pulgar pequeño (flecha roja), y cuatro cojinetes; C: pie con cinco dedos y plantar con seis cojinetes.

Figure 2. A: First specimen of $A$. jelskii collected in Chile, it correspond to adult male (CZZA030). B: Left hand with five fingers, with a small thumb (red arrow), and four pads; C: foot with five toes and plantar with six pads. 
falta de exploraciones biológicas y catastros de la fauna de vertebrados en el norte de Chile, lugar que alberga las ecoregiones menos estudiadas del país (Valladares 2012), lo que queda en evidencia junto con la descripción de la nueva especie Eligmodontia dunaris (Spotorno et al. 2013) sumando dos especies al listado de los mamíferos de Chile en los últimos años.

\section{AGRADECIMIENTOS}

Agradecemos al Servicio Agrícola y Ganadero (SAG) por el permiso de captura de micromamíferos $\mathrm{N}^{\circ} 306$, Enero 2013 y Febrero 2014. A la Corporación Nacional Forestal (CONAF) por el permiso para hacer investigaciones científicas en las Áreas Silvestres Protegidas. A Eduardo Palma (PUCSSUC), Guillermo D'Elia (IEEUACU) y Ángel Spotorno (LCM) por compartir datos de colectas de roedores de la Región de Arica y Parinacota. Agradecemos también a Roberto Tancara Querquezana por entregarnos fotografías del ejemplar vivo y la localidad de su hallazgo. Este estudio fue financiado por el proyecto Universidad de Tarapacá / UTA Mayor de Investigación Científica y Tecnológica, Proyecto N 4710 - 2015.

\section{BIBLIOGRAFÍA}

Anderson, S. 1997. Mammals of Bolivia, taxonomy and distribution. Bulletin of American Museum of Natural History 231:1-652.

DíAz, M.M., \& BÁrquEZ, R.M. 2007. Los mamíferos silvestres de la Provincia de Jujuy, Argentina: sistemática y distribución. In: The Quintessential Naturalist: Honoring the Life and Legacy of Oliver P. Pearson (Eds. Kelt, D.A., Lessa, E.P., Salazar-Bravo, J. \& J.L. Patton), pp. 417-578. California: University of California Publications in Zoology 134.

FErro, L.I. 2010. Micromamíferos del noroeste argentino: gradientes altitudinales y la transición bosque-punamonte. Unpublished $\mathrm{PhD}$ thesis, Universidad Nacional de
Tucumán, Tucumán, Argentina. 181 pp.

Galliari, C.A., Pardiñas, U.F.J., \& Gain, F.J. 1996. Lista comentada de los mamíferos de argentinos. Mastozoología Neotropical 3(1):39-6.

JAyat, J.P., Ortiz, P.E., \& González, R. 2013. First record of Abrothrix jelskii (Thomas, 1894) (Mammalia: Rodentia: Cricetidae) in Salta province, northwestern Argentina: Filling gaps and distribution map. Check List 9(4):902905.

Martínez, O., Pérez, M.E., Taucer, E., \& Rechberger, J. 2009. Fauna vertebrada de San Cristóbal en el altiplano sur de Bolivia. Kempffiana 5(1):28-55.

Pacheco, V., Cardenillas, R., Salas, E., Tello, C., \& Zeballos, H. 2009. Diversidad y endemismo de los mamíferos del Perú. Revista Peruana de Biología 16(1):005-032.

Salazar - Bravo, J., Tarifa, T., Aguirre, L.F., Yensen, E., \& Yates, T.L. 2003. Revised Checklist of Bolivian Mammals. Occasional Papers of Museum of Texas Tech University 220:1-28.

SAnBorn, C. 1947. Geographical races of the rodent Akodon jelskii Thomas. Fieldiana Zoology 31(17):133-142.

Smith, M.F., \& Patton, J.L. 1991. Variation in Mitochondrial Cytochrome b Sequence in Natural Populations of South American Akodontine Rodents (Muridae: Sigmodontinae). Molecular Biology and Evolution 8(1):85-103.

Spotorno, A.E., Zuleta, C., Walker, L.I., Manriquez, G., VAlladares, P., \& Marín, J.C. 2013. A small, new gerbilmouse Eligmodontia (Rodentia: Cricetidae) from dunes at the coasts and deserts of north-central Chile: molecular, chromosomic, and morphological analyses. Zootaxa 3683(4):377-394.

Tarifa, T., \& Yensen, E. 2001. Mamíferos de los bosques de Polylepis de Bolivia. Revista Boliviana de Ecología 9:2944.

Teta, P., Pardiñas, U.F.J., \& D`Elia, G. 2006. “Abrotrichinos”. In: Mamíferos de Argentina: sistemática y distribución (Eds. Bárquez, R.M., Díaz, M.M. \& R.A. Ojeda), pp. 192197. Tucumán: Sociedad Argentina para el Estudio de los Mamíferos.

Valladares, P. 2012. Mamíferos terrestres de la Región de Atacama, Chile. Comentarios sobre su distribución y estado de conservación. Gayana 76(1):13-28.

Recibido: 04.06.14

Aceptado: 18.05.15 